\title{
Topological bifurcations in a model society of reasonable contrarians
}

\author{
Franco Bagnoli* \\ Dipartimento di Fisica e Astronomia, Università di Firenze, \\ Via G. Sansone 1, 50017 Sesto Fiorentino (FI), Italy; \\ also INFN, sez. Firenze. \\ Raúl Rechtman† \\ Instituto de Energías Renovables, \\ Universidad Nacional Autónoma de México, \\ Apdo. Postal 34, 62580 Temixco Mor., México
}

\begin{abstract}
People are often divided into conformists and contrarians, the former tending to align to the majority opinion in their neighborhood and the latter tending to disagree with that majority. In practice, however, the contrarian tendency is rarely followed when there is an overwhelming majority with a given opinion, which denotes a social norm. Such reasonable contrarian behavior is often considered a mark of independent thought, and can be a useful strategy in financial markets.

We present the opinion dynamics of a society of reasonable contrarian agents. The model is a cellular automaton of Ising type, with antiferromagnetic pair interactions modeling contrarianism and plaquette terms modeling social norms. We introduce the entropy of the collective variable as a way of comparing deterministic (mean-field) and probabilistic (simulations) bifurcation diagrams.

In the mean field approximation the model exhibits bifurcations and a chaotic phase, interpreted as coherent oscillations of the whole society. However, in a one-dimensional spatial arrangement one observes incoherent oscillations and a constant average.

In simulations on Watts-Strogatz networks with a small-world effect the mean field behavior is recovered, with a bifurcation diagram that resembles the mean-field one, but using the rewiring probability as the control parameter. Similar bifurcation diagrams are found for scale free networks, and we are able to compute an effective connectivity for such networks.
\end{abstract}

\section{INTRODUCTION}

Social norms are the basis of a community. They are often adopted and respected even if in contrast with an agent's immediate advantage, or, alternatively, even if they are costly with respect to a naive behavior. Indeed, the social pressure towards a widespread social norm is sometimes more powerful than a norm imposed by punishments.

It is well known that the establishment of social norms is a difficult task and their imposition is not always fulfilled. This problem has been affronted by Axelrod in a game-theoretic formulation [1], as the foundation of the cooperation and of the society itself. Axelrod's idea is that of a repeated game. Although in a one-shot game it is always profitable to win not following any norm, in a repeated game there might be several reasons for cooperation [2, the most common ones are direct reciprocity and reputation. In all these games, the crucial parameters are the cost of cooperation with respect to defeat, and the expected number of re-encounters with one's opponent or the probability that one's behavior will become public. One can assume that these aspects are related to the size of the local community with which one interacts and the fraction of people in this community that share the acceptance of the social norm. Indeed, the behav-

\footnotetext{
* franco.bagnoli@unifi.it

$\dagger$ rrs@cie.unam.mx
}

ior of a spatial social game is strongly influenced by the network structure 3].

In the presence of a social norm, people can manifest a conformist or a non conformist or contrarian attitude, characterized by the propensity to agree or disagree with the average opinion in their neighborhood.

Contrarian agents were first discussed in the field of finance 4 and later in opinion formation models 5 . Contrarian behavior may have an advantage in financial investment. Financial contrarians look for mispriced investments, buying those that appear to be undervalued by the market and selling those that are overpriced. In opinion formation models, contrarians gather the average opinion of their neighbors and choose the opposite one. Reasonable contrarians do not violate social norms, i.e., they agree with conformists if the majority of neighbors is above a certain threshold.

Models of social dynamics have been studied extensively. 6]. In this paper we model the dynamics of a homogeneous community with different degrees of reasonable contrarianism.

One of the main motivations for this study is that of exploring the possible behavior of autonomous agents employed in algorithmic trading in an electronic market. Virtually all markets are now electronic [7] and the speed of transaction require the use of automatic agents (algorithmic trading) 8]. Our study can be consider as an exploration of possible collective effects in a homogeneous automatic market.

We consider a simplified cellular automaton model [9]. 
Each agent can have one of two opinions at time $t$, and we study the parallel evolution of such agents, which can be seen also as a spin system.

A society of conformists can be modeled as a ferromagnet and one of contrarians as an antiferromagnet. Each agent changes his opinion according to the local social pressure or what is the same, the average opinion of his neighbors. In spin language, social norms can be represented as plaquette terms since they are non additive and important when the social pressure is above or below given thresholds.

The model, presented in Sec. II, simulates a society of $N$ reasonable contrarians that can express one of two opinions, 0 and 1 . At each time step, each agent changes its opinion according to a transition probability that takes into account the average opinion of his neighbors, that is, the local social pressure and the adherence to social norms. The neighborhoods are fixed in time. The transition probability depends on a parameter $J$, analogous to the spin coupling in the Ising model, which is positive for a society of conformists (ferromagnet) and negative for one of contrarians (antiferromagnet).

For a one dimensional society where the neighborhood of each agent includes its first $k$ nearest neighbors, $k$ is the connectivity, the average opinion fluctuates around the value $1 / 2$, regardless of the values of the parameters of the transition probability. Simulations of the onedimensional version of the model show irregular fluctuations at the microscopic level, with short range correlations 10].

The mean field approximation of the model for the average opinion is a discrete map which exhibits bifurcation diagrams as the parameters $k$ and $J$ change, as discussed in Sec. III. The diagrams show a period doubling route towards chaos.

In Sec. IV we discuss the model on Watts-Strogatz networks that exhibit the small-world effect [11. We find a bifurcation diagram as the fraction $p$ of rewired links changes. Since the opinion of agents change probabilistically, we speak of probabilistic bifurcation diagrams.

In Sec. V, the reasonable nonconformist opinion model is extended to scale-free networks. Again, we observe a probabilistic bifurcation diagram, similar to the previous ones, by varying the coupling $J$. We are able to obtain a good mapping of the scale-free parameters onto the mean field approximation with fixed connectivity $k$.

In order to compare the deterministic and probabilistic bifurcation diagrams, we exploit the entropy $\eta$ of the average opinion. In the deterministic case, large values of $\eta$ correspond to positive values of the Lyapunov exponent. In Secs. III, IV and V we show that $\eta$ can be used to characterize numerically order and disorder in deterministic and probabilistic bifurcation diagrams. Finally we present some conclusions.

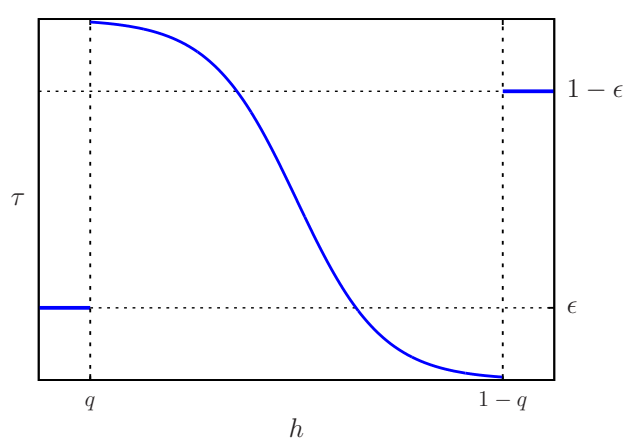

FIG. 1. (Color online) The transition probability $\tau(h)$ given by Eq. (3) with $J=-3, k=20, q=0.1$, and $\varepsilon=0.2$.

\section{THE MODEL}

Each of the $N$ agents has opinion $s_{i}(t)$ at the discrete time $t$ with $s_{i} \in\{0,1\}$ and $i=0, \ldots, N-1$. The state of the society is $\boldsymbol{s}=\left(s_{0}, \ldots, s_{N-1}\right)$. In the context of cellular automata and discrete magnetic systems, the state at site $i$ is $s_{i}$ and the spin at site $i$ is $\sigma_{i}=2 s_{i}-1$ respectively. The average opinion $c$ is given by

$$
c=\frac{1}{N} \sum_{i} s_{i} .
$$

The opinion of agent $i$ evolves in time according to the opinions of his neighbors, identified by an adjacency matrix with components $a_{i j} \in\{0,1\}$. If agent $j$ is a neighbor of agent $i, a_{i j}=1$, otherwise $a_{i j}=0$. The adjacency matrix defines the network of interactions and is considered fixed in time. The connectivity $k_{i}$ of agent $i$ is the size of his neighborhood,

$$
k_{i}=\sum_{j} a_{i j} .
$$

The average local opinion or social pressure $h_{i}$, is defined by

$$
h_{i}=\frac{\sum_{j} a_{i j} s_{j}}{k_{i}} .
$$

The opinion of agent $i$ changes in time according to the transition probability $\tau\left(s_{i} \mid h_{i}\right)$ that agent $i$ will hold the opinion $s_{i}$ at time $t+1$ given the local opinion $h_{i}$ at time $t$. This transition probability, shown in Fig. 1, is given by

$$
\tau(h)= \begin{cases}\varepsilon & \text { if } h<q, \\ \frac{1}{1+\exp (-2 J(2 h-1))} & \text { if } q \leq h \leq 1-q, \\ 1-\varepsilon & \text { if } h>1-q,\end{cases}
$$

with $\tau(h)=\tau(1 \mid h)$. The quantity $q$ denotes the threshold for the social norm, and $\varepsilon$ the probability of being 
reasonable. With $\varepsilon=0$ or $q=0, s=(0, \ldots, 0)$ and $\boldsymbol{s}=(1, \ldots, 1)$ are absorbing states [10]. In the following we set $\varepsilon=0.2$ and $q=0.1$ if not otherwise stated. The results are qualitatively independent of $\varepsilon$ and $q$ as long as they are small and positive. The transition probability $\tau$ has the symmetry

$$
\tau(1-h)=1-\tau(h)
$$

With $J>0$ and $q<h_{i}<1-q$, agent $i$ will likely agree with his neighbors, a society of conformists. With $J<0$ and $q<h_{i}<1-q$, agent $i$ will likely disagree with his neighbors, a contrarian society. For $0 \leq h \leq q$ or $1-q \leq h \leq 1$ agent $i$ will likely agree (if $\varepsilon$ is small) with the majority of his neighbors, regardless of the value of $J$.

We might also add an external field $H$, modeling news and broadcasting media, but in this study we always keep $H=0$. We are thus modeling a completely uniform society, i.e., we assume that the agent variations in the response to stimuli are quite small. Moreover, we do not include any memory effect, so that the dynamics is completely Markovian.

In the language of spin systems, $\tau\left(h_{i}\right)$ is the transition probability of the heat bath dynamics of a parallel Ising model with ferromagnetic, $J>0$, or antiferromagnetic, $J<0$, interactions [12. The behavior of the transition probability in the regions $h<q$ and $h>1-q$ may be seen as due to a non-linear plaquette term that modifies the ferro/antiferro interaction. If we set $\varepsilon=0$ and $J=-\infty$, the system becomes deterministic (in magnetic terms, this is the limit of zero temperature).

In one dimension, with $k=3,1 / 3<q \leq 1 / 2$ and $\varepsilon=0$ this model exhibits a nontrivial phase diagram, with two directed-percolation transition lines that meet a first-order transition line in a critical point, belonging to the parity conservation universality class 13 . In this case, we have the stability of the two absorbing states for $J>0$ (conformist society or ordered phase), while for $J<0$ (anti-ferro or contrarian) the absorbing states are unstable and a new, disordered active phase is observed. The model has been studied in the one-dimensional case with larger neighborhood 10. In this case one observes again the transition from an ordered to an active, microscopically disordered phase, but with no coherent oscillations. Indeed, if the system enters a truly disordered configuration, then the local field $h$ is everywhere equal to 0.5 and the transition probabilities $\tau$ become insensitive to $J$ and equal to 0.5 , see Eq. (3).

\section{MEAN FIELD APPROXIMATION}

The simplest mean-field description of the model is given by

$$
c^{\prime}=f(c)=\sum_{w=0}^{k}\left(\begin{array}{l}
k \\
w
\end{array}\right) c^{w}(1-c)^{k-w} \tau(w / k),
$$

(a)

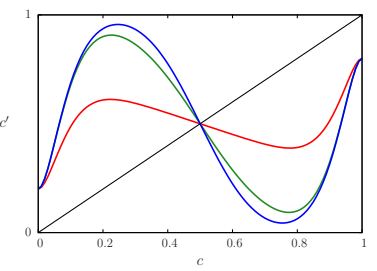

(b)

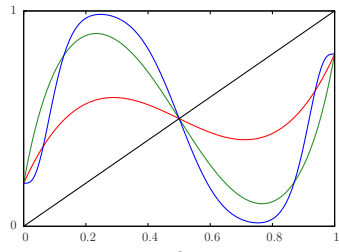

FIG. 2. (Color online) (a) Graphs of the mean field map, Eq. (5) for different values of $J$ and $k=20$. From bottom to top for $c<1 / 2, J=-0.5$ (red), $J=-3.0$ (green), and $J=-6.0$ (blue). (b) Graphs of Eq. (5) for different values of $k$ and $J=-6$. From bottom to top for $c \sim 0.2, k=4$ (red), $k=10$ (green), and $k=38$ (blue).

with $c^{\prime}=c(t+1)$ and $c=c(t)$ [14]. The term in parenthesis on the r.h.s of this expression denotes the $w$-combinations from a set of $k$ elements. In Fig. 2 we show some graphs of $f$. The map $f$ has the same symmetry property as the transition probabilities $\tau$,

$$
f(1-c)=1-f(c) .
$$

The mean-field map, Eq (5), shows a bifurcation diagram when varying the parameter $J$ (Figs. 3 (a) and 4 (a)). Since the mean-field map is deterministic, these bifurcations can be characterized by means of the Lyapunov exponent $\lambda$. However, in order to study these diagrams and that produced by the stochastic microscopic simulations on the same ground, let us introduce the Boltzmann entropy [15 $\eta$ of the collective variable $c$. This entropy is a good measure of disorder and in the case of deterministic maps, large values of $\eta$ correspond to positive values of the Lyapunov exponent as we show below. For probabilistic processes, it is a measure of disorder. We define the normalized Boltzmann entropy $\eta$ as

$$
\eta=\frac{-1}{\log L} \sum_{i=1}^{L} q_{i} \log q_{i}
$$

where the interval $[0,1]$ is divided in $L$ disjoint intervals $I_{i}$ of equal size (bins) and $q_{i}$ is the probability that $c \in I_{i}$, $i=0, \ldots, L-1$. It is clear that $0 \leq \eta \leq 1$, the lower bound corresponding to a fixed point, the upper one to the uniform distribution $q_{i}=1 / L$. The probabilities $q_{i}$ are found numerically by finding the fraction of time one orbit visits the bin $I_{i}$.

The map $f$ of Eq. (5) depends on the parameters $J$, $k, q$ and $\varepsilon$. We keep $q$ and $\varepsilon$ fixed. By changing $J$ for $k=20$ we find the bifurcation diagram shown in Fig. 3 (a) with the corresponding values of the Lyapunov exponent $\lambda$ and the entropy $\eta$ in Fig. 3 (b). The bifurcation diagram appears to show a period-doubling cascade, but it is more complex than that. For $0>J \geq J_{0}$ there are period-one orbits and for $J_{0}>J \geq J_{1}$ period-two orbits. For $J_{1}>J \geq J_{2}$ the orbits appear to have period 
(a)

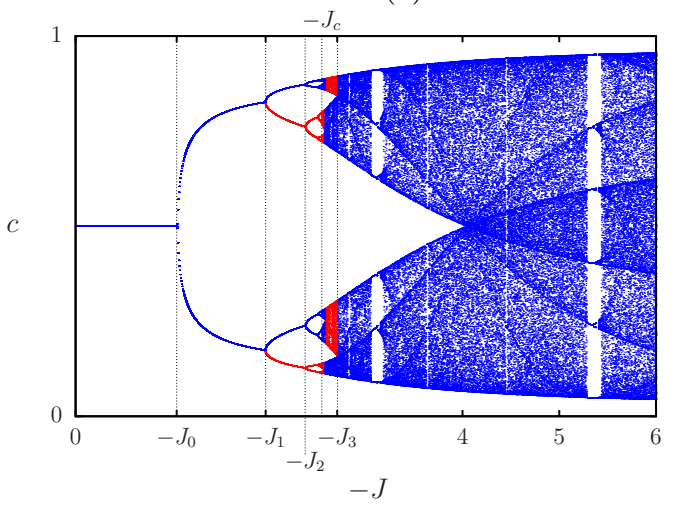

(b)

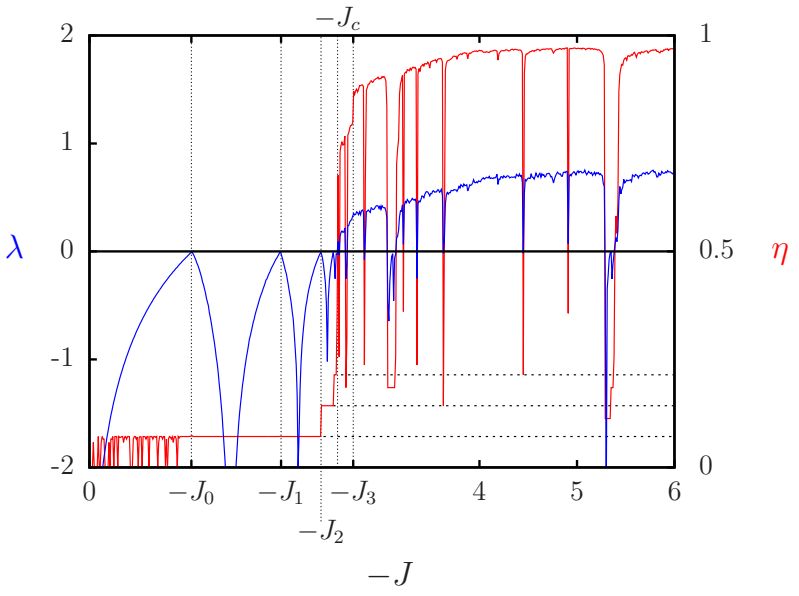

FIG. 3. (Color online) (a) Bifurcation diagram of the mean field map, Eq. (5), by varying $J$. The doubling bifurcation route to chaos ends at $J=J_{c}$. For $0>J \geq J_{2}$ and $J_{3}>J \geq 6$ there is only one attractor (blue dots). For $J_{2}>J \geq J_{c}$ there are two, one of them corresponds to the lower branches that bifurcate up to $J_{c}$ (red dots), and the other one to the upper branches (blue dots). For $J_{c}>J \geq J_{3}$ there are two chaotic attractors, one corresponding to the lower branches (blue dots), the other to the top branches (red dots). For every value of $J$, the dots are 64 iterates of the map of Eq. (5) after a transient of $10^{3}$ time steps. For values of $J$ with only one basin of attraction the orbits do not depend on the initial average opinion $c(t=0)$. For values of $J$ that correspond to two attractors, one of them was found with $c(0)=0.1$, the other one with $c(0)=0.9$. (b) The Lyapunov exponent $\lambda$, top curve on the left of the graph (in blue), and entropy $\eta$, top curve on the right of the graph (in red), For every value of $J, \lambda$ was evaluated during $10^{3}$ time steps. The entropy $\eta$ is computed using $L=2^{14}$ bins. After a transient of 500 time steps, the probability distribution was evaluated during the next $100 \times L$ time steps. The horizontal dotted lines are drawn, starting from below, at $\eta=w / m, w=1, \ldots, 3$ corresponding to periodic orbits of period $2^{w}$. The connectivity is $k=20$ and the vertical dotted lines are drawn at $J_{0}=-1.045$, $J_{1}=-1.965, J_{2}=-2.375, J_{c}=-2.545$, and $J_{3}=-2.705$. (a)

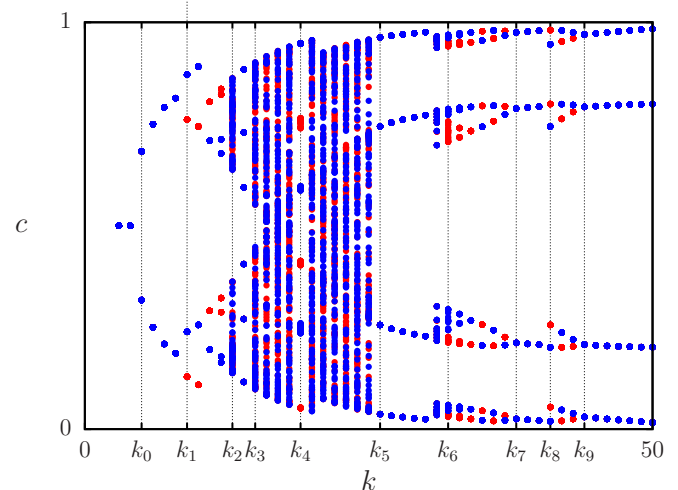

(b)

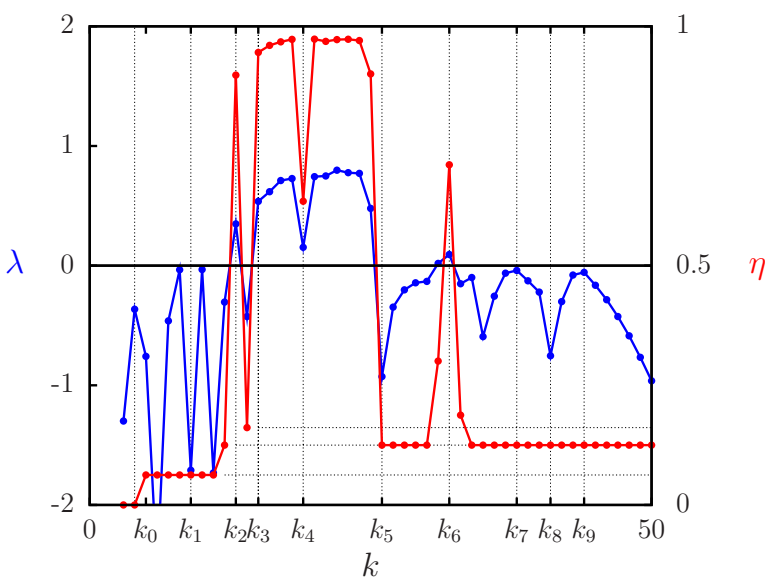

FIG. 4. (Color online.) (a) Bifurcation diagram of the mean field map of Eq. (5) varying $k$ for $J=-6$. For every value of $k$, two initial values were considered, $c(0)=0.1$ and $c(0)=0.9$, and for each one 64 iterations were plotted after a transient of $10^{3}$ time steps. For $k<k_{0}$ there is a fixed point and for $k_{0} \leq k<k_{1}$ period-two orbits. For $k_{1} \leq k<k_{2}$ the bottom branches (in red) correspond to one attractor and the top branches (in blue) to the other one. For $k_{3} \leq k<k_{5}$ the orbits are chaotic but for $k=k_{4}$ there are two attractors, one (in red) corresponds to the alternate clusters of points starting from below, the other one (in blue) to the other three clusters of points. For $k_{6} \leq k<k_{7}$, and $k_{7} \leq k<k_{8}$ there are again two attractors, one cluster (in red) corresponds roughly to the bottom branches and the other one (in blue) to the top branches. These attractors are not chaotic except for $k=k_{6}$. (b) The Lyapunov exponent $\lambda$, top curve for $k<k_{2}$ (in blue), and the entropy $\eta$, top curve for $k_{3}<k<k_{5}$ (in red), both as functions of the connectivity $k$ for the same values of $J$ as in (a). For each value of $k, \lambda$ was evaluated during $10^{3}$ time steps. For $\eta, L=2^{16}$ and the probability distribution was evaluated during the $100 \times L$ time steps after a transient of $10^{3}$ time steps. The horizontal dotted lines correspond, starting from below, to perio-two, period-four, and period-six orbits. The vertical dotted lines are drawn at $k_{0}=5, k_{1}=9$, $k_{2}=13, k_{3}=15, k_{4}=19, k_{5}=26, k_{6}=32, k_{7}=38$, $k_{8}=41$, and $k_{9}=44$. 
four, but actually correspond to two separate period-two attractors. In other words, for $J=J_{1}$ there is a pitchfork bifurcation. For $J_{2}>J \geq J_{c}$ there are two separate period-doubling bifurcations with the appearance of chaos at $J=J_{c}$. For $J_{c}>J \geq J_{3}$ there are two chaotic attractors that merge at $J=J_{3}[16$. Due to the symmetry of the map, Eq. (6), if $c$ belongs to one of the basins of attraction, $1-c$ belongs to the other one.

In Fig. 4 (a) we show the bifurcation diagram of the map $f$ as $k$ changes with fixed $J, \varepsilon$, and $q$. For $k<k_{0}$ there are period-one orbits and for $k_{0} \leq k<k_{1}$ periodtwo orbits. For $k_{1} \leq k<k_{2}$ there are two period-two attractors. The two attractors are again present for $k=$ $k_{4}, k_{6} \leq k<k_{7}$, and for $k_{8} \leq k<k_{9}$. For $k=k_{4}$ and $k=k_{6}$ the two attractors are chaotic. In Fig. 4 (b) we show $\lambda$ and $\eta$ as $k$ changes. Again, chaotic orbits have entropy larger than $\eta_{c}=1 / 2$. Chaotic orbits are present for $k=k_{2}, k_{3} \leq k \leq k_{5}$, and $k=k_{6}$. Both bifurcation diagrams, Figs. 3 (a) and 4 (a) are symmetric around $c=0.5$, a consequence of the symmetry of the mean field map, Eq. (6).

In Figs. 5 (a) and (b) we show the phase diagrams, as $J$ and $k$ change, of the Lyapunov exponent $\lambda$ and the entropy $\eta$ respectively. In (a) the points correspond to $\lambda>0$ and in (b) to $\eta>\eta_{c}=1 / 2$. These figures show that both quantities are a good measure of chaos in this case. The values of $\lambda$ and $\eta$ shown in Fig. 3 (b) correspond to those on the vertical line $k=20$ of Fig. 5 (a) and (b) respectively. The results shown in Fig. 4 (b) correspond to the horizontal lines $J=-6$ of Fig. 5 (a) and (b).

\section{SMALL-WORLD NETWORKS}

In the Watts-Strogatz small-world network model there is a smooth change from a regular to a random lattice [11. Starting with a network with $N$ agents, where the neighborhood of each agent is formed by his $k$ nearest neighbors, with probability $p$ each neighbor is replaced by another individual chosen at random. We call $p$ the long-range connection probability. In Fig. 6 we show the return map of the average opinion $c$, after a long transient, together with the mean field return map $f$ of Eq. (5) for several values of $p$. For $p=0$, the density $c$ fluctuates around its mean value 0.5 . As $p$ grows, the system becomes more homogeneous and the distribution of points approaches the mean field behavior, even though the mean field approximation has been derived by imposing the absence of correlations. As shown in the figure, for $p=0.6$ the return map is already close to the mean field behavior and for $p=1$ it is indistinguishable from it.

We show in Figs. 77(a) and (b) the probabilistic bifurcation diagrams of $c$ as a function of the probability of long-range connections $p$ for $J=-6$ and $J=-3$ and the same value of $k$. In both figures, for $0<p \lesssim p_{0}$ and $p_{0}<p \lesssim p_{1}$ we can identify period-one and period-two orbits respectively. For $p_{1} \lesssim p \lesssim p_{2}$ there are two period- (a)

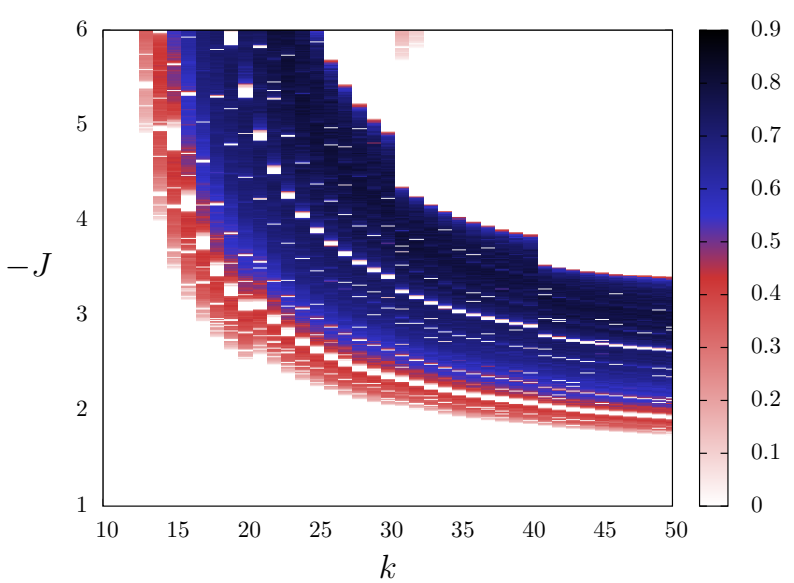

(b)

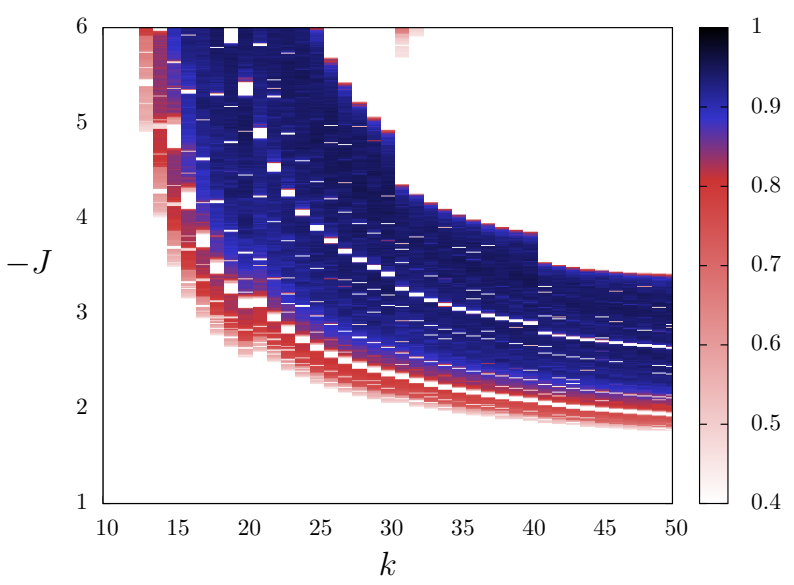

FIG. 5. (Color online) (a) Phase diagram showing positive values the Lyapunov exponent $\lambda$ for the mean field approximation Eq. (5) as a function of $k$ and $J$. For each value of $J$ and $k$ the Lyapunov exponent $\lambda$ was calculated during $10^{3}$ time steps. (b) Phase diagram of the entropy $\eta$ showing values larger than $1 / 2$, for the same values of $\varepsilon$ and $q$ as in (a). After a transient of $2 \cdot 10^{3}$ time steps the probability distribution was evaluated during the next $10^{3}$ time steps on $L=128$ bins.

two attractors which become indistinguishable for $p \simeq p_{2}$. For $p_{2} \lesssim p$ there is only one attractor. In Figs. 7(b) and (c) we show the corresponding entropy. We would like to find a threshold $\eta_{d}$ for the appearance of disorder, similar to $\eta_{c}$ of the mean field approximation, and we propose $\eta_{d}=\eta\left(p_{2}\right)$ shown as the horizontal lines in Figs. 7

In Fig. 8 (a) and (b) we show the phase diagrams of the entropy $\eta$ for $p=0.5$ and $p=1$ respectively. It is evident that for $p=1$, Fig. 8 (a), the diagram is very similar to that of Fig. 5. while for $p=0.5$, Fig. 8 (a), there is a sort of dilatation of the high-entropy region, extending to larger values of $k$ (and beginning also with higher values of $k$. The dependence on $J$ is much less marked. It is possible to roughly understand these results assuming 
(a)

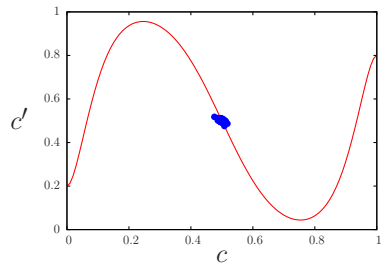

(c)

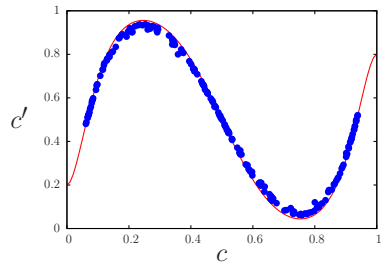

(b)

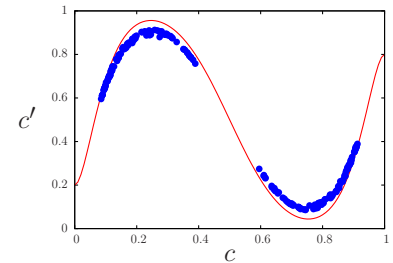

(d)

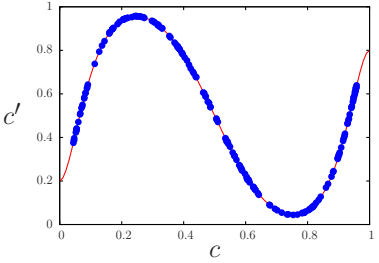

FIG. 6. (Color online) Return map of the average opinion $c$ on small-world networks for several values of the long-range connection probability $p$ with $J=-6, k=20, N=10^{3}$, and a transient of $10^{3}$ time steps. The following 200 iterations are shown as (blue) dots. The (red) continuous curve is Eq. (5). (a) $p=0.0$, (b) $p=0.5$, (c) $p=0.6$, and (d) $p=1.0$. (a)

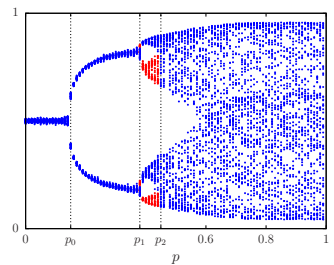

(c)

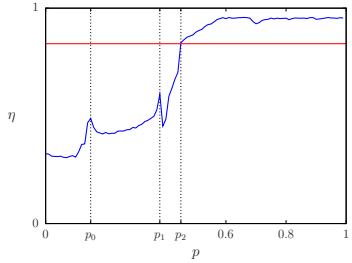

(b)

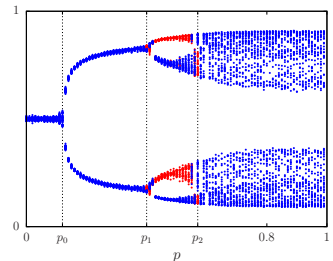

(d)

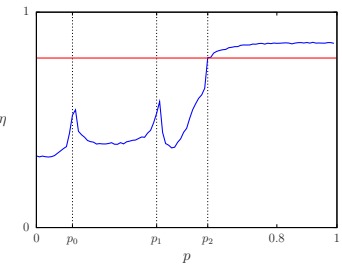

(a)

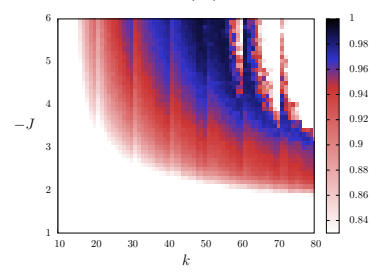

(b)

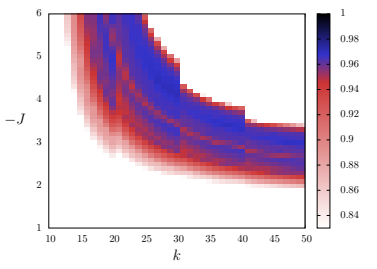

FIG. 8. (Color online) Entropy phase diagrams of simulations on small-world networks as functions of $k$ and $J$ for (a) $p=0.5$ and (b) $p=1.0$. The colored region corresponds to $\eta>\eta_{d}=0.8$. Entropy computed with 128 bins, lattice size $N=10^{4}$, sampling time $1.2 \cdot 10^{4}$ steps after a transient of $4 \cdot 10^{3}$ steps.

that the main contributions to the mean-field character of the collective behavior come from the fraction of links that are rewired (long-range connections), that depends on $p$.

\section{SCALE-FREE NETWORKS}

Human and technological networks often present a scale-free character, with different degrees of correlation among nodes. In this section we present results of the model on uncorrelated scale-free networks [17. Starting from a fully connected group of $m$ agents, other $N-m$ agents join sequentially, each one choosing $m$ neighbors among those already in the group. The choice is preferential, the probability that a new member chooses agent $i$ is proportional to its connectivity $k_{i}$, the number of neighbors agent $i$ already has. Another way of building the network is choosing a random edge of a random node and connecting to the other end of the edge, since such an edge arrives to a vertex with probability proportional to $k p(k)[18$.

In the Appendix we show that the model dynamics on scale-free networks is comparable to the mean field approximation of Sec. III on a network with constant connectivity $k$ with

$$
k=\alpha m, \quad \alpha \sim 1.7 .
$$

bifurcation diagrams as functions of the long range probability $p$. For $p \lesssim p_{0}$ there are almost periodic orbits of period one and for $p_{0} \lesssim p \lesssim p_{1}$ of period two. For $p_{1} \lesssim p \lesssim p_{2}$ we find two attractors, one (in red) in the lower branches, the other one (in blue) in the top ones. (c) and (d) The entropy $\eta$ as a function of $p$. The (red) lines mark the value of $\eta_{d}$. (a) and (c) $J=-6, p_{0} \sim 0.15, p_{1} \sim 0.38, p_{2} \sim 0.45$, and $\eta_{d}=\eta\left(p_{2}\right)=0.835$. (b) and (d) $J=-3, p_{0} \sim 0.12, p_{1} \sim 0.40$, $p_{2} \sim 0.57$, and $\eta_{d}=\eta\left(p_{2}\right)=0.787$. In (a) and (b) the number of agents is $N=5 \cdot 10^{4}$, the connectivity is $k=20$. After a transient of $4 \cdot 10^{3}$ time steps, the probability distribution is evaluated using $L=256$ bins during the next $100 \times L$ time steps.
In Figs. 9 (a) and (b) we show the probabilistic bifurcation diagrams of the model on scale-free networks as a function of $J$ for two values of $m$ and in Figs. 9 (c) and (d) we show the bifurcation diagram of the mean field approximation, Eq. (5), for the corresponding values of $k$ according to Eq. (8). We find a qualitative agreement between these bifurcation diagrams.

In Figs. 9 (e) and (f) we show the entropy of of the mean field approximation and of the simulations on scalefree networks. We find a reasonable agreement when $\eta>\eta_{d}$ with $\eta_{d}=\eta\left(J_{0}\right)$ with $J_{0}$ the value of $J$ for which the entropy of the mean field approximation crosses the line $\eta=1 / 2$ for the first time. Thus, the entropy is a 
(a)

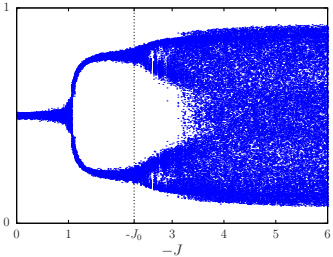

(c)

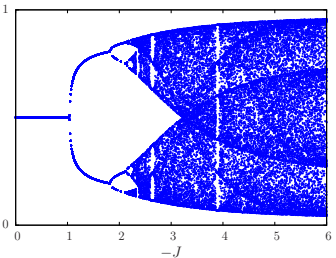

(e)

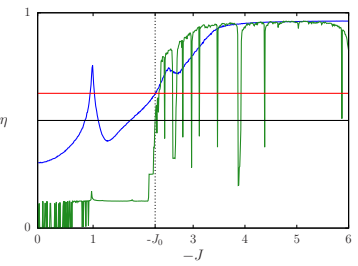

(b)

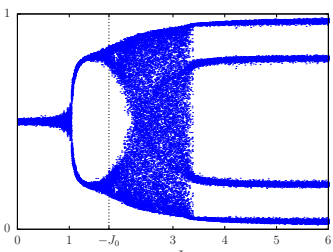

(d)

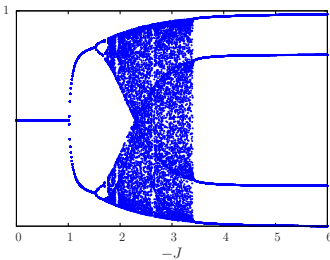

(f)

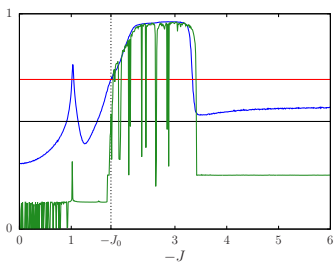

FIG. 9. (Color online) Comparisons between simulations on scale-free networks and mean-field approximation. In (a) and (b) bifurcation diagrams of simulations on scale-free networks with $m=15$ and $m=30$, respectively. In both cases, the number of agents is $N=10,000$ and for every value of $J$, the initial opinions are chosen at random with $c=1 / 2$, and 128 values of $c$ are plotted after a transient of 300 time steps. In (c) and (d) we show the bifurcation diagrams of the mean field approximation with $k=25 \sim 1.7 \times 15$ and $k=51 \sim 1.7 \times 30$, respectively. In both figures, $c(0)$ is chosen at random and 128 values of $c$ are plotted after a transient of $10^{3}$ time steps. In (e) and (f) the entropy $\eta$ of the simulations on scale-free networks (top curve - blue for $J>-1$ ) is compared with that of the mean-field approximation, (bottom curve - green for $J>-1$ ). In (e), $k=15$ and $m=25$, in (f) $k=30$ and $m=51$. The entropy is found by dividing the unit interval in 256 bins. For each value of $J, c(0)=0.1$ and after a transient of $10^{3}$ time steps the entropy was evaluated during the following $2.56 \cdot 10^{4}$ time steps. In (a) and (e) $J_{0}=-2.267$, and in (b) and (f) $J_{0}=-1.766$.

good way of comparing both dynamics when $k$ and $m$ are related according to Eq. (8). Above $\eta_{d}$, both entropies are numerically similar, except where there are periodic windows in the mean field approximation, and this agreement is better for $m=30$ and $k=51$.

\section{CONCLUSIONS}

We studied a reasonable contrarian opinion model. The reasonableness condition forbids the presence of absorbing states. In the model, this condition depends on two parameters that are held fixed. The model also depends on the connectivity $k$ which may vary among agents, and the coupling parameter $J$. The neighborhood of each agent is defined by an adjacency matrix that can have fixed or variable connectivity (fixed or power-law) and a regular or stochastic character.

The interesting observable is the average opinion $c$ at time $t$. We computed the entropy $\eta$ of the stationary distribution of $c$, after a transient.

In the simplest case, the neighborhood of each agent includes $k$ random sites. In this case, the mean field approximation for the time evolution of the average opinion exhibits, by changing $J$, a period doubling bifurcation cascade towards chaos with an interspaced pitchfork bifurcation. A positive (negative) Lyapunov exponent corresponds to an entropy larger (smaller) than $\eta_{c}=1 / 2$. Thus, entropy is a good measure of chaos for this map, and can be also used in the simulations of the stochastic microscopic model.

The bifurcation diagram of the mean field approximation as a function of $k$ shows periodic and chaotic regions, also with a pitchfork bifurcation. Again, entropies larger than $\eta_{c}$ correspond to chaotic orbits.

Actual simulations on a one-dimensional lattice show incoherent local oscillations around $c=1 / 2$. By rewiring at random a fraction $p$ of local connections, the model presents a series of bifurcations induced by the smallworld effect: the density $c$ exhibits a probabilistic bifurcation diagram that resembles that obtained by varying $J$ in the mean field approximation. These smallworld induced bifurcations are consistent with the general trend, long-range connections induce mean field behavior. This is the first observation of this for a system exhibiting a chaotic mean field behavior. Indeed, the small-world effect makes the system more coherent (with varying degree). We think that this observation may be useful since many theoretical studies of population behavior have been based on mean field assumptions (differential equations), while actually one should rather consider agents, and therefore spatially-extended, microscopic simulations. The well-stirred assumption is often not sustainable from the experimental point of view. However, it may well be that there is a small fraction of long-range interactions (or jumps), that might justify the small-world effect.

The model on scale-free networks with a minimum connectivity $m$ shows a similar behavior to that of the mean field approximation of the model on a network with constant connectivity $k$, Eq. (5) if $k=\alpha m$ with $\alpha \sim 1.7$.

In summary, we have found that, as usual, long-range rewiring leads to mean-field behavior, which can become chaotic by varying the coupling or the connectivity. Similar scenarios are found in actual microscopic simulations, also by varying the long-range connectivity, and in scale free networks.

This study can have applications to the investigation of collective phenomena in algorithmic trading. 


\section{ACKNOWLEDGEMENTS}

Interesting discussions with Jorge Carneiro and Ricardo Lima are acknowledged. This work was partially supported by Recognition Project UE grant $\mathrm{n}^{\circ} 257756$ and project PAPIIT-DGAPA-UNAM IN109213.

\section{APPENDIX}

The similarity between the bifurcations diagrams in Figs. 3 (a) and 4 (a), which comes out from the similarities of the mean-field maps when changing $J$ and $k$ (Fig. 2), can be explained by using a continuous approximation for the connectivity $k$. By using Stirling's approximation for the binomial coefficients in Eq. (5), for intermediate values of $c$ [19], we obtain

$$
\left(\begin{array}{l}
k \\
w
\end{array}\right) c^{w}(1-c)^{k-w} \simeq \frac{1}{\sqrt{2 \pi k c(1-c)}} \exp \left[\frac{-k(w / k-c)^{2}}{2 c(1-c)}\right]
$$

In this approximation, Eq. (5) can be written as

$$
c^{\prime}=\int_{-\infty}^{\infty} \mathrm{d} x \sqrt{\frac{k}{2 \pi c(1-c)}} \exp \left[-\frac{k(x-c)^{2}}{2 c(1-c)}\right] \tau(x)
$$

with $x$ the continuous approximation of $w / k$. This expression is just a Gaussian convolution of $\tau$, i.e., a smoothing of the transition probability, as can be seen by comparing Fig. 1 with Fig. 2. This smoothing has the effect of reducing the slope of the curve in a way similar to changing $J$ (but is depends also on $c$ ), and this explains the similarities between the bifurcation diagrams in Fig. 3 (a) and 4 (a). For instance, Fig. 3 (a) is obtained for $k=20$, a value that in Fig. 4 (a) corresponds to a chaotic strip just after a window with six branches. A similar window can be observed also in Fig. 3 (a) by increasing $J$ from the value $J=-6$ of Fig. 4 (a).

This approximation can be used also to find the "effective" connectivity of the model on a scale-free network. The mean field approximation for a non-homogeneous network can be written as

$$
c_{k}^{\prime}=\sum_{\substack{s_{1}, s_{2}, \ldots, s_{k} \\ j_{1}, j_{2}, \ldots, j_{k}}} \prod_{i=1}^{k} c_{j_{i}}^{s_{i}}\left(1-c_{j_{i}}\right)^{1-s_{i}} Q\left(j_{i} \mid k\right) \tau\left(h_{i}\right),
$$

with $c_{k}^{\prime}$ the probability that the opinion of an agent with connectivity $k$ at time $t+1$ is one, and $c_{j}$ the probability that the opinion of an agent with connectivity $j$ at time $t$ is one. The sum on the r.h.s is taken over the opinions $s_{1}, \ldots, s_{k}$ of the $k$ agents in the neighborhood, and over their connectivities $j_{1}, \ldots, j_{k}$. The variables $s_{i}$ take the values zero or one, while $j_{i}$ ranges from $m$ to $\infty$. The quantity $Q(j \mid k)$ is the probability that the agent with connectivity $j$ is connected to another one of connectivity $k$ and $\sum_{j} Q(j \mid k)=1$.

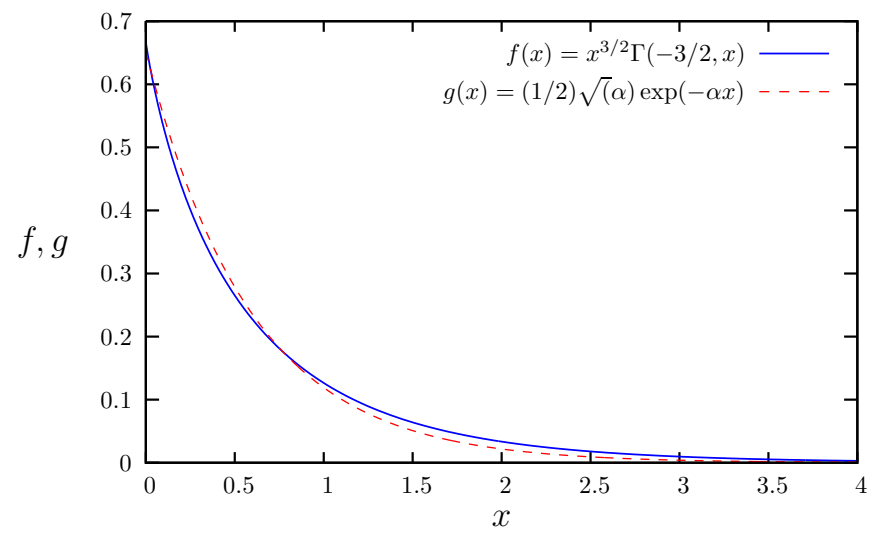

FIG. 10. (Color online) Comparisons between the two functions $f(x)$ ((blue) solid line) and $g(x)$ ((red) dashed line), for $\alpha=1.7$, corresponding to the minimum of $\int_{0}^{4}(f(x)-g(x))^{2} \mathrm{~d} x$

Since this network is symmetric, $k Q(j \mid k) P(k)=$ $j Q(k \mid j) P(j)$ (detailed balance). It is also nonassortative, so $Q(j \mid k)$ does not depend on $k$ and we can write $Q(j \mid k)=\phi(j)$. By summing the detailed balance condition over $j$ we get $\phi(k)=k P(k) /\langle k\rangle$. Therefore, Eq. (11) becomes

$c_{k}^{\prime}=\sum_{s_{1}, s_{2}, \ldots, s_{k}} \tau\left(\frac{\sum_{i} s_{i}}{k}\right) \prod_{i=1}^{k} \sum_{j_{i}} \frac{j_{i} P\left(j_{i}\right)}{\langle k\rangle} c_{j_{i}}^{s_{i}}\left(1-c_{j_{i}}\right)^{1-s_{i}}$.

In the previous equation, $s_{i}$ is either zero or one, so that only one between $c_{j_{i}}^{s_{i}}$ and $\left(1-c_{j_{i}}\right)^{1-s_{i}}$ is different from zero. Assuming that $c_{k}$ depends only slightly on $k$ in Eq. 12, we approximate $\left(\sum_{j_{i}} j_{i} P\left(j_{i}\right) c_{j_{i}}\right) /\langle k\rangle$ with $c$ and we get

$$
\begin{aligned}
c_{k}^{\prime} & =\sum_{s_{1}, s_{2}, \ldots, s_{k}} \tau\left(\frac{\sum_{i} s_{i}}{k}\right) \prod_{i=1}^{k} c^{s_{i}}(1-c)^{1-s_{i}} \\
& =\sum_{w} \tau\left(\frac{w}{k}\right)\left(\begin{array}{c}
k \\
w
\end{array}\right) c^{w}(1-c)^{k-w}
\end{aligned}
$$

with $w=\sum_{i} s_{i}$. In order to close the equation we average $c_{k}^{\prime}$ over the probability distribution $P(k)$.

By using the approximation of Eq. (9), we get

$$
\begin{aligned}
c^{\prime} & =\sum_{k=m}^{\infty} P(k) \sum_{w} \tau\left(\frac{w}{k}\right)\left(\begin{array}{c}
k \\
w
\end{array}\right) c^{w}(1-c)^{k-w} \\
\simeq & \int_{m}^{\infty} \mathrm{d} k P(k) \\
& \quad \int_{-\infty}^{\infty} \mathrm{d} x \tau(x) \sqrt{\frac{k}{2 \pi c(1-c)}} \exp \left[-\frac{k(x-c)^{2}}{2 c(1-c)}\right],
\end{aligned}
$$

where $x=w / k$. For scale-free networks the connectivity 


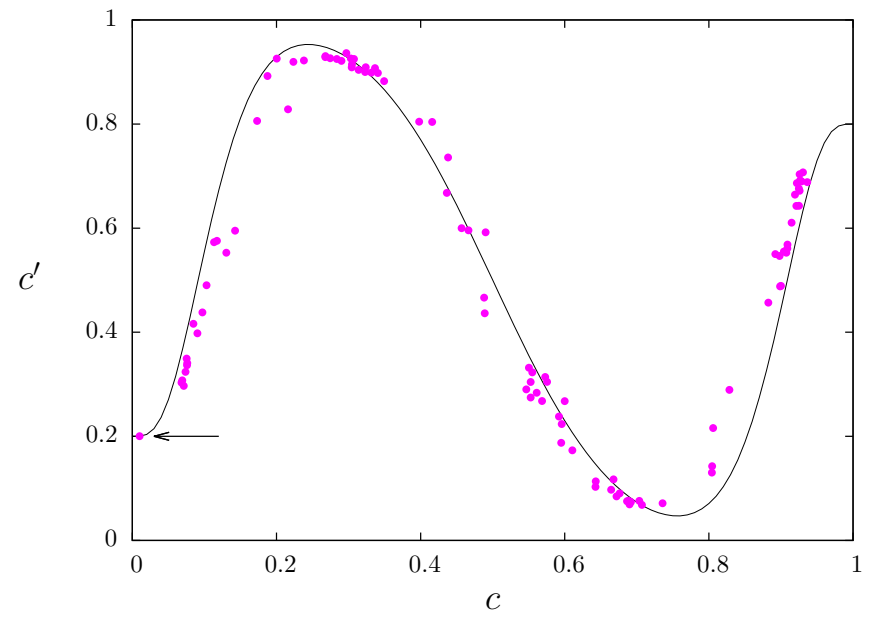

FIG. 11. (Color online) First 100 steps of the return map for the density $c$ of the model on a scale free network with $N=10^{4}, m=20, J=-4$. The first iterate is marked by the arrow. The continuous curve is the graph of Eq. 13 with $k=34$

distribution $P$ is given by $P(k)=2 m^{2} k^{-3}$. Then a

$$
\begin{aligned}
c^{\prime} & \simeq \int_{-\infty}^{\infty} \mathrm{d} x \frac{2 m^{2} \tau(x)}{\sqrt{(2 \pi c(1-c))}} \int_{m}^{\infty} \mathrm{d} k k^{-5 / 2} \exp (-k A) \\
& =\int_{-\infty}^{\infty} \mathrm{d} x \frac{m^{1 / 2} \tau(x)}{(2 \pi c(1-c))^{1 / 2}} 2(m A)^{3 / 2} \Gamma\left(-\frac{3}{2}, m A\right)
\end{aligned}
$$

where $A=A(x)=(x-c)^{2} /(2 c(1-c))$ and $\Gamma(a, x)$ is the incomplete upper gamma function extended to negative values of $a$ (the function $x^{-a} \Gamma(a, x)$ is single-valued and analytic for all values of $a$ and $x$ [20]).

The function $f(y)=y^{3 / 2} \Gamma(-3 / 2, y)$ is well approxi- mated by $g(y)=(1 / 2) \sqrt{\alpha} \exp (-\alpha y)$, as shown in Fig. 10 . Therefore we can write

$$
c^{\prime} \simeq \int_{-\infty}^{\infty} \mathrm{d} x \tau(x) \sqrt{\frac{\alpha m}{2 \pi c(1-c)}} \exp \left[-\frac{\alpha m(x-c)^{2}}{2 c(1-c)}\right] .
$$

This last expression has the form of Eq. (10), with an effective connectivity $\tilde{k}=\alpha m$.

Since the argument $y$ of $g(y)$ is $m A(x)=m(x-$ $c)^{2} /(2 c(1-c))$, the substituted $g(x)$ results to be a Gaussian, centered around $x=c$. The important values of $g(x)$ lie between 0 and 4, depending on the value of $c$. In this interval, the best approximation of $f(y)$ (the minimum of $\int_{0}^{4}\left(f(y)-g(y)^{2} \mathrm{~d} y\right)$ is around $\alpha \simeq 1$.7. Therefore $\tilde{k}$ is definitively different from the average connectivity $\langle k\rangle=2 m$.

In conclusions, also in the case of a non-assortative scale-free network, the probability of getting a site with value 1 in the mean field approximation is given by

$$
c^{\prime}=\sum_{j=0}^{\tilde{k}} c^{j}(1-c)^{\tilde{k}-j} \tau\left(\frac{j}{\tilde{k}}\right)
$$

with $\tilde{k} \simeq 1.7 \mathrm{~m}$.

As usual, the mean field predictions are only approximately followed by actual simulations. In Fig. [11 we show the first 100 steps of the return map of the density $c$ for $J=-0.4$. The scale-free network is fixed, with $m=20, N=10,000$ and the initial opinions of the agents are chosen at random with $c=0.01$. The arrow marks the first point, that follows the mean field prediction with $\alpha=1.7(\tilde{k}=34)$, as in Fig. 11 , but then, due to correlations, the return maps follows a different curve. This implies that nontrivial correlations establish also in scale-free networks.
[1] R. Axelrod, The Evolution of Cooperation (Basic Books, New York 1984).

[2] M. Nowak, Science 314, 1560 (2006).

[3] K. Klemm, V. M. Eguiluz, R. Toral and M. San Miguel, Phys. Rev. E 67, 026120 (2003).

[4] A. Corcos, J.-P. Eckmann, A. Malaspinas, Y. Malevergne, D. Sornette, Quantitative Finance 2264 (2002).

[5] S. Galam, Physica A, 333453 (2004).

[6] C. Castellano, S. Fortunato and V. Loreto, Rev. Mod. Phys. 81, 591 (2009).

[7] P. Jain, Journal of Finance 60, 2955-2985 (2005).

[8] A. Chaboud, B. Chiquoine, E. Hjalmarsson and C. Vega, Journal of Finance, Forthcoming; FRB International Finance Discussion Paper No. 980. (2013). http://dx.doi.org/10.2139/ssrn.1501135

[9] F. Bagnoli, F. Franci, and R. Rechtman, in Cellular Automata, edited by S. Bandini, B. Chopard, and M. Tomassini (Springer-Verlag, Berlin, 2002), p. 249.

[10] F. Bagnoli, F. Franci and R. Rechtman, Phys. Rev. E 71, 046108 (2005).
[11] D.J. Watts and S.H. Strogatz, Nature 393, 409 (1998).

[12] B. Derrida, in Fundamental Problem in Statistical Mechanics, edited by H. van Beijeren (Elsevier, New York, 1990), Vol. VII., pp. 276.

[13] F. Bagnoli, N. Boccara, and R. Rechtman, Phys. Rev. E 63, 046116 (2001).

[14] A. Ilachinski, Cellular Automata: A Discrete Universe, (World Scientific, 2001), p. 352 .

[15] L. Boltzmann, Vorlesungen über Gastheorie, Leipzig, J. A. Barth,; Partt I, 1896, Part II, 1898. English translation by S. G. Brush, Lectures on Gas Theory, University of California Press, 1964, Chapter I, Sec. 6.

[16] H. E. Nusse and J. A. Yorke, Physica D 57, 39 (1992). H. E. Nusse, E. Ott, and J. A. Yorke, Phys. Rev. E 49, 1073 (1994). V. Avrutin, M. Schanz, Phys. Rev. E 70026222 (2004).

[17] L. Barabási, R. Albert, Science 286509 (1999).

$[18]$

[19] E. Ott, Chaos in Dynamical Sysytems, Cambridge University 
[20] M. Abramowitz and I. Stegun, Handbook of Mathematical
Functions, Government Printing Office, also (NY, Dover, 1985). pp. 260-261 